\title{
ENHANCEMENT OF SPECIALISED VERTICAL TURNING LATHE ACCURACY THROUGH MINIMISATION OF THERMAL ERRORS DEPENDING ON TURNING AND MILLING OPERATIONS
}

\author{
M. Mares ${ }^{1 *}$, O. Horejs ${ }^{1}$ \\ ${ }^{1}$ Czech Technical University in Prague, Faculty of Mechanical Engineering, Department of Production Machines and \\ Equipment (RCMT), Prague, Czech Republic \\ *Corresponding author; e-mail: m.mares@rcmt.cvut.cz
}

\begin{abstract}
Achieving high workpiece accuracy is a long-term goal of machine tool designers. There are many causes of workpiece inaccuracy, with thermal errors being the most dominant. Indirect compensation (using predictive models) is a promising strategy for reducing thermal errors without increasing machine tool cost. A modelling approach using thermal transfer functions (a dynamic method with a physical basis) embodies the potential to deal with this issue. The method does not require interventions into the machine tool structure, uses a minimum of additional gauges and its modelling and calculation speed is suitable for real-time applications with fine results with up to $80 \%$ thermal error reduction. Advanced machine tool thermal error compensation models have been successfully applied on various kinds of single-purpose machines (milling, turning, floor-type, etc.) and implemented directly into their control systems. This research reflects modern trends in machine tool usage and as such is focused on the applicability of the modelling approach to describe specialised vertical turning lathe versatility. The specialised vertical turning lathe is adequately capable of carrying out turning and milling operations. Calibration of the reliable compensation model is a real challenge. The applicability of the approach during immediate switching between turning and milling operations is discussed in more detail.
\end{abstract}

\section{Keywords:}

Thermal error; Compensation; Accuracy; Machine tool; Milling; Turning

\section{INTRODUCTION}

The heat generated e.g. by moving axes and machining processes creates thermal gradients, resulting in the thermal elongation and bending of machine tool (MT) elements, which substantially deteriorate MT accuracy. Consequently, up to $75 \%$ of all geometrical errors of machined workpieces are caused by temperature effects [Mayr 2012]. Thermal errors could be sufficiently reduced by new design MT concepts which are less sensitive to thermal effects. This type of intervention in the MT structure leads to a pareto set of different parameters and designers have to concentrate on preserving other MT properties as well [Grossmann 2015]. At the same time, redesigning MT structure is usually possible in the prototype phase of new products. Adaptive or intelligent control of cooling systems [Hellmich 2018], integrated additional sensors in MT structure [Naumann 2018] or direct (in-process) measurement techniques [Zimmermann 2020] could also be very efficient in minimising thermo-mechanical impacts on MT accuracy, but they do increase machine and operation costs and result in machining process interruptions and prolonged production time. A very promising contemporary approach is the use of FEM coupled with model order reduction (MOR) techniques to reduce computing time [Hernandez-Becerro 2018]. This solution still encounters the problem of boundary condition complexity at the machine or component level. In contrast, indirect (software) compensation of thermal errors at the tool centre point (TCP) is one of the most widely employed reduction techniques due to its cost-effectiveness and ease of application.

Ordinarily, approximation models are based on measured auxiliary variables [Brecher 2004] (temperature, spindle speed, etc.) used to calculate the resulting thermally induced displacements at the TCP. Many strategies have been investigated to establish these models, e.g. multiple linear regressions (MLR) [Srinivas 2017], artificial neural networks (ANN) [Mize 2000], transfer functions (TF) [Brecher 2004], etc. The majority of the compensation models introduced in the literature have the potential to significantly reduce MT thermal errors. The methods differ in the amount and type of input variables, training and modelling time required for composition and model structure architecture (white, black or grey-boxes [Li 2008]). Therefore, further efforts should be focused on the 
applicability and verification of approaches in real industrial conditions and environments.

A thermal error modelling approach for a specialised vertical turning lathe capable of turning and milling operations is proposed in this research (a model concept for a different versa machine - a turning-milling center - was introduced in [Mares1 2020]). The target machine was placed in a real industrial environment and the existing compensation model of thermal effects caused by turning operations [Mareš2 2020] was extended to include a description of milling operations. The extended compensation model based on TFs was directly implemented into the MT control system, and internal information from the MT control system (temperatures measured close to heat sources used originally for diagnostic and safety purposes) and a parameter containing the workpiece clamping diameter are used as the only model inputs. The approach was verified practically during simultaneous activity of both technological processes according to the ISO 230-3 international measuring conditions standard [ISO 230-3 2007]. Moreover, the compensation model considers spatial thermal deformations over the entire table area. The measuring fixture that was developed and the analysis of thermal error compensation are presented in more detail.

\section{TARGET MACHINE}

A multi-functional machine fully combines at least two production technologies [Moriwaki 2008]. The target machine for this article is a double-column specialised vertical turning lathe capable of full-fledged turning and milling operations. The carousel table is active during the turning operations and the spindle acts as the cutting tool support and vice versa, i.e. the table only positions and the spindle, equipped with the milling tool, becomes active during milling operations.

The target machine was placed in a real industrial environment, namely a MT manufacturer's production hall. The hall is a non-air-conditioned huge space divided into several naves each equipped with a row of production machines. Despite the place character, the environment could be considered as stable regarding also manufacturer's years of experience. A schema of the MT with highlighting of the approximate positions of the temperature probes used further in modelling effort is depicted in Fig. 1.

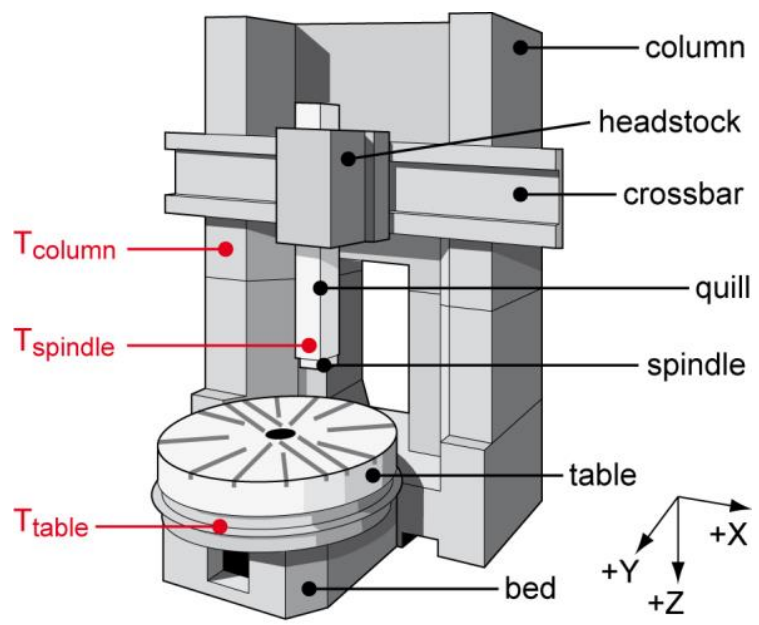

Fig. 1: Schema of the target machine - a specialised vertical turning lathe - with approximate positions of temperature probes.
The input temperatures (spindle front bearing temperature $T_{\text {spindle, }}$ rotary table bearing temperature $T_{\text {table }}$ and reference column temperature $T_{\text {column}}$ ) were taken directly from MT control system. Their positions were chosen close to the examined thermal sources. The primary purpose of the temperature sensors is diagnostic of component wear.

\section{MODELLING OF MACHINE TOOL THERMO- MECHANICAL BEHAVIOUR AFFECTED BY TURNING AND MILLING OPERATIONS}

The research aims to construct a compensation model of thermal errors caused by both technological operations which enables resolution of their synergistic activity. Thus, it pertains to the case where the output of the thermomechanical system (the measured deformation between the table and the TCP) is affected by both turning and milling.

The thermal error model is divided into two parts. The first part approximates thermal errors caused by turning operations. This part is the subject of a publication [Mareš2 2020] in which the calibration process of thermal errors depending on table rotation speed and workpiece clamping diameter is described in detail. The model was successfully verified under finishing cutting conditions. The second part of the model approximates the errors caused by spindle rotation. The general modelling approach, calibration, and TF identification process of the second independent part of the model will be described in the following sections.

\subsection{Modelling approach based on transfer functions}

The concept behind the modelling approach lies in usage of a minimum of additional gauges (the only information from the MT control system [Brecher 2004]), an open structure that is easy to extend and modify (advantageous for machine learning principles and intelligent solutions within the MT [Blaser 2019]), real time application and ease of implementation into the MT control systems. A set of TFs appears to be a suitable tool.

The compensation strategy based on TFs is a dynamic method with a physical basis. A discrete TF is used to describe the link between the excitation and its response:

$y(t)=u(t) \cdot \varepsilon+e(t)$,

$y(t)=\frac{a_{n} Z^{-n}+\cdots+a_{1} Z^{-1}+a_{0} Z^{0}}{b_{m} Z^{-m}+\cdots+b_{1} Z^{-1}+b_{0} Z^{0}} u(t) ;$ where $m>n$.

The vector $u(t)$ in equations (1) and (2) is the TF input in the time domain, $y(t)$ is the output vector in the time domain, $\varepsilon$ represents the TF in the time domain, $e(t)$ is the disturbance value (further neglected), $a_{n}$ is the calibration coefficient of the TF input, $b_{m}$ is the calibration coefficient of the TF output, $n$ is the order of the TF numerator, $m$ is the order of the TF denominator, and $z$ is a complex number.

The differential form of the TF (generally suitable for programming languages like Python) is introduced in equation (3),

$$
\begin{aligned}
y(k)= & \frac{u(k-n) a_{n}+\cdots+u(k-1) a_{1}+u(k) a_{0}}{b_{0}}- \\
& -\frac{y(k-m) b_{m}+\cdots+y(k-1) b_{1}}{b_{0}},
\end{aligned}
$$

where $k-n(k-m)$ signifies the $n$-multiple ( $m$-multiple) delay in sampling frequency. Linear parametric models of autoregressive with external input (ARX) or outputs error $(\mathrm{OE})$ identifying structures are used with the help of Matlab 
Identification Toolbox [Ljung 2020]. The ARX as optimal model structure (with the best fitting quality and robustness) is discussed in [Mayr 2018] where MISO (multiple input single output) models handing with arbitrary TCP measurements are introduced.

Excitations in the case of the employed TFs mean temperatures measured close to heat sinks or sources and the responses stand for the linear deflections in the examined directions. The approximation quality of the simulated behaviour is expressed by a local peak-to-peak approach (differences of maximum and minimum simulated or measured deformations) and a global approach based on the least square method (equation (4)).

$f i t=\left(1-\frac{\left\|Z_{\text {mea. }}-Z_{\text {sim. }}\right\|}{\left\|Z_{\text {mea. }}-\bar{Z}_{\text {sim. }}\right\|}\right) \cdot 100$

The $Z_{\text {mea }}$ value in equation (4) is the measured output (thermal displacements in the Z-direction), $Z_{\text {sim. }}$ is the simulated/predicted model output, and $\bar{Z}_{\text {mea }}$. expresses the arithmetic mean of the measured output over time.

\subsection{Calibration test set-up and conditions within the MT milling configuration}

Data was acquired during the experiments using the $\mathrm{NI}$ cRIO-9014 produced by National Instruments and LabVIEW software. The NI cRIO-9014 collected NC data from the machine tool control system via OPCUA communication protocol.

All of the results and conclusions are associated with the following experiment conditions. The model was valid for no-load or finishing conditions. The model describing spindle thermo-mechanical impact was calibrated in one MT axial configuration (at the centre of the table), and compensation was realised in linear directions only.

Eddy current sensors firmly held in the measuring fixture were employed for noncontact sensing of the displacements between the TCP (in the directions $X$ and $Z$ shown in Fig. 2) represented by a test mandrel (length 125 $\mathrm{mm}$, diameter $40 \mathrm{~mm}$ ) and the table (regular position of the workpiece), per international standard [ISO 230-3 2007]. The displacements were sensed in micrometre resolution.

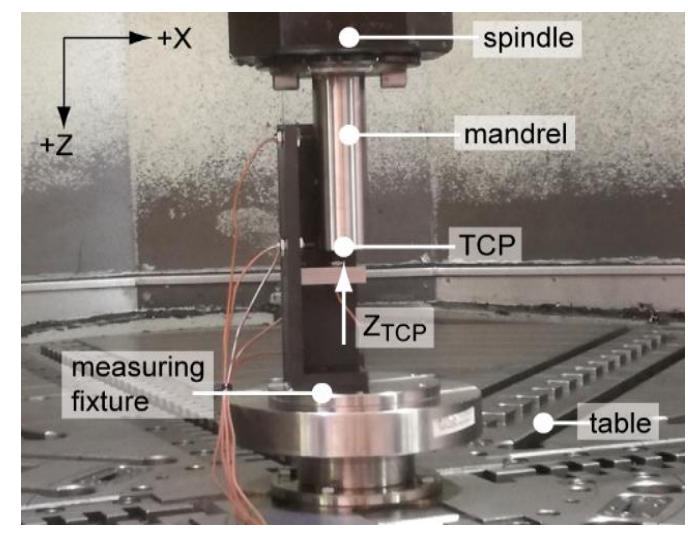

Fig. 2: Experimental set-up for calibration measurement of spindle speed influence on thermal error.

All relevant temperatures were recorded from the MT control system in $0.1 \mathrm{~K}$ resolution. The behaviour of temperature inputs into the thermo-mechanical system in relative coordinates (a suitable form for TFs) along with spindle speed set-up during the calibration test are shown in Fig 3.

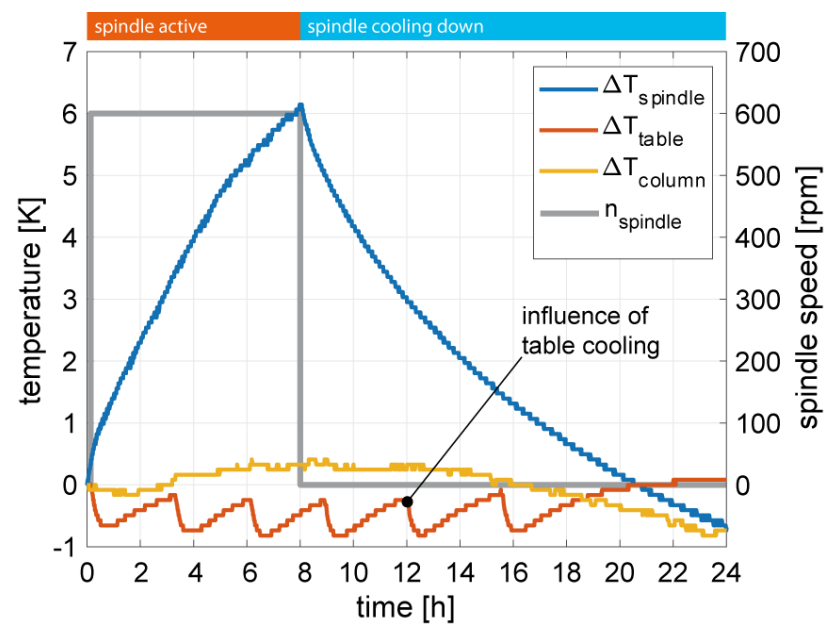

Fig. 3: Calibration test set-up, conditions and thermomechanical system input behaviour.

\subsection{Identification of model approximating thermal errors caused by spindle speed}

Data processing and TF identification, as well as MT thermal behaviour modelling, were performed in Matlab and Matlab Simulink (version R2017a).

The effort is focused on minimising thermal errors in the most affected $Z$ direction influenced by coupling effects of spindle and table rotations. Compensations in other directions could follow the similar mechanisms.

The calibration measurement consisted of a part of transient behaviour between two thermodynamic equilibria (the MT in approximate balance with its surroundings and the MT steady state during heat source activity) ended after one work shift (8 hours). The measurement of the complete transient behaviour would have taken very long time with indistinct benefits in this case. The behaviour is caused by lack of spindle cooling. The TF identification process of spindle activity is shown in Fig. 4 . The graph shows the following outputs: measured, simulated and residual (the difference between measured and simulated values representing the MT compensated state). A linear dependency between measured input and output values was observed during both the heating and the cooling phases. Therefore, only the heating (first 8 hours from Fig. 3) was considered in the TF identification process.

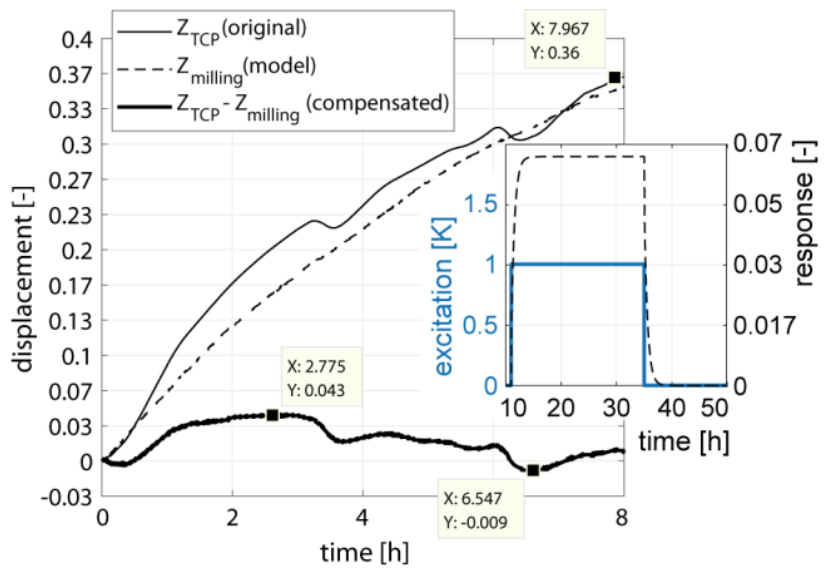

Fig. 4: Measured and simulated outputs from the thermomechanical system during the calibration test and step response of the identified TF. 
The TF model of thermal deformations in the $Z$ direction between the TCP and the table for the MT milling configuration is expressed by equation (5).

$Z_{\text {milling }}=\left(\Delta T_{\text {spindle }}-\Delta T_{\text {column }}\right) \cdot \varepsilon_{1}$.

$Z_{\text {milling }}$ in equation (5) is the simulated output from the thermo-mechanical system (displacement), $\Delta T_{\text {spindle }}$ is the spindle bearing temperature expressed in relative coordinates taken directly from the MT control system, $\Delta T_{\text {column. }}$ represents information in relative coordinates regarding changes in ambient temperature, and $\varepsilon_{1}$ is the identified TF in the time domain. The stability of the identified TF $\varepsilon 1$ and a better understanding of spindle activity in the MT thermo-mechanical behaviour are expressed by the LTI step response depicted in the right part of Fig. 4. System excitation represents the sudden change of the difference $\Delta T_{\text {spindle }}-\Delta T_{\text {column }}=1 \mathrm{~K}$ (the blue curve in the graph), and system response is the predicted deformation given by equation (5) (the black dashed curve in the graph). The established calibration coefficients $a_{n}$ and $b_{\mathrm{m}}$ of the identified TF are summarised in Tab. 1. The order of the TF was selected based on the best fit value. The fit value attained by identified model form equation (5) during the calibration test is $75 \%$. Expressed by the peak-to-peak the original deformation state of the MT was reduced 7-fold.

Tab. 1: Coefficients of the identified TF describing spindle rotation influence on thermal error in the $Z$ direction.

\begin{tabular}{ccccc}
\hline TF & \multicolumn{4}{c}{ Coefficients } \\
\hline & $\boldsymbol{a}_{0}$ & $\boldsymbol{a}_{1}$ & $\boldsymbol{a}_{2}$ & $\boldsymbol{a}_{3}$ \\
$\boldsymbol{\varepsilon}_{1}$ & 10.01220 & -9.9895457 & 0 & 0 \\
& $\boldsymbol{b}_{0}$ & $\boldsymbol{b}_{1}$ & $\boldsymbol{b}_{2}$ & $\boldsymbol{b}_{3}$ \\
& 1 & -0.877647 & 0.020108 & -0.141865 \\
\hline
\end{tabular}

It is assumed that the approximation model of thermal errors caused by spindle rotation is position-independent.

\subsection{Thermal error approximation model considering machine tool's capability of turning and milling operations}

An approximation model of thermal errors caused by table rotation (turning operations) was established in [Mareš2 2020] and is expressed by the following equation:

$Z_{\text {turning }}=\left[\left(\Delta T_{\text {table }}-\Delta T_{\text {column }}\right) \cdot \varepsilon_{2}\right] \cdot g$,

where $Z_{\text {turning }}$ is the simulated output from the thermomechanical system, $\Delta T_{\text {table }}$ is the table bearing temperature difference, $\varepsilon_{2}$ represents the TF approximating thermal errors due to table rotation (for TF calibration coefficients see $\mathrm{Tab} .2$ ) and $g$ represents the gain factor dependent on the workpiece clamping diameter. The approximation model of thermal errors caused by table rotation is positiondependent.

Tab. 2: Coefficients of the TF describing table rotation influence on thermal error in the $Z$ direction (taken from [Mareš2 2020]).

\begin{tabular}{ccccc}
\hline TF & \multicolumn{4}{c}{ coefficients } \\
\hline & $\boldsymbol{a}_{0}$ & $\boldsymbol{a}_{1}$ & $\boldsymbol{a}_{2}$ & $\boldsymbol{a}_{3}$ \\
$\varepsilon 2$ & -82.41672 & 82.41479 & 0 & 0 \\
& $\boldsymbol{b}_{0}$ & $\boldsymbol{b}_{1}$ & $\boldsymbol{b}_{2}$ & $\boldsymbol{b}_{3}$ \\
& 1 & -0.64533 & 0.10375 & -0.45835 \\
\hline
\end{tabular}

The thermal error approximation model considering machine tool's capability of turning and milling operations is given by superposition of the two components: the model of thermal errors caused by spindle speed (equation (5)) and the model of thermal errors caused by table rotation (equation (6)). The resultant approximation model is expressed by equation (8)

$$
\begin{aligned}
& Z_{\text {coupled }}=Z_{\text {milling }}+Z_{\text {turning }}, \\
& Z_{\text {coupled }}=\left(\Delta T_{\text {spindle }}-\Delta T_{\text {column }}\right) \cdot \varepsilon_{1}+ \\
& \quad+\left[\left(\Delta T_{\text {table }}-\Delta T_{\text {column }}\right) \cdot \varepsilon_{2}\right] \cdot g,
\end{aligned}
$$

where $Z_{\text {coupled }}$ is the simulated output from the thermomechanical system considering MT's capability of turning and milling operations. The compensation is realised in a control system through an offset setup on the MT linear axis. The model (equation (8)) is implemented directly into the MT control system via SIMATIC STEP7 (TIA Portal), the programming language for Siemens. A diagnostic screen arranges communication with the MT operator and provides visualisation of model input/output parameters with optional corrective intervention.

\section{MODEL VERIFICATION TEST CONSIDERING SUBSEQUENT TURNING AND MILLING OPERATIONS}

The basic premise is minimum interaction of thermomechanical system inputs (measured temperatures) during coincidental activity of calibrated thermal sources wherein the output (the measured deformation between the table and the TCP) is affected.

\subsection{Measuring fixture}

The measuring fixture from Fig. 2 was extended to measure deformations in the whole table area (on table diameters $D=0, D=1500$ and $D=3000 \mathrm{~mm}$ ) as illustrated in Fig. 5 . Mounting a thermal stable frame (composite rods with a 'zero' thermal expansion coefficient equipped with displacement probe holders) to the spindle stator and the measuring artefacts to the table enabled the measuring fixture extension to record deformations during spindle and table activity without interruption. The composite material of the thermal stable frame is chosen to avoid hanging in $Z$ machine direction caused by thermal transfer from the spindle and to ensure reliable measurement of thermal errors in $X$ machine direction (not part of this paper).

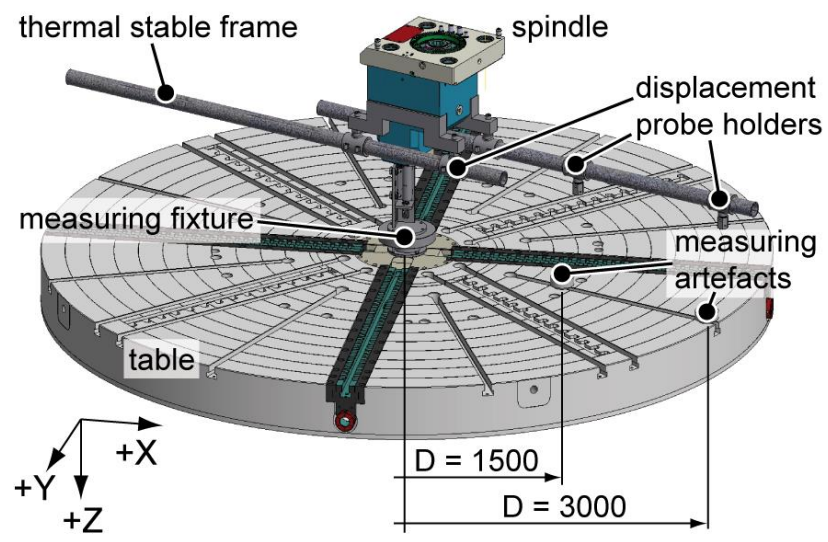

Fig. 5: Illustration of extended measuring fixture to thermal stable frame ready for thermal error measurement within independent spindle and table activity. 
The mounted extended measuring fixture in the MT workspace is shown in Fig. 6. The fixture is captured in the measuring location. Contactless displacement probes fixed in magnetic holders sense additional thermal errors between the frame and measuring artefacts placed on the table. The extended measuring fixture makes it possible to obtain four measured outputs from the thermo-mechanical system: $Z_{\mathrm{TCP}}, Z_{\mathrm{D}=0}, Z_{\mathrm{D}=15000}$ and $Z_{\mathrm{D}=3000}$.

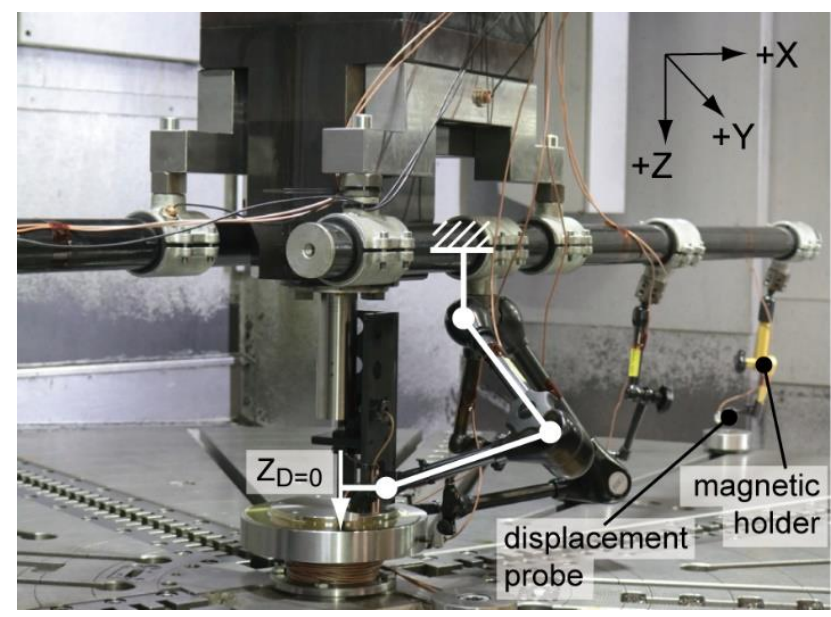

Fig. 6: Extended measuring fixture placed in the MT workspace in the measuring location.

\subsection{Verification test set-up}

The table and the spindle speed spectra set-ups (followed by cooling phases) during the verification test are shown in Fig. 7. The table was positioned regularly during its activity in the measuring location for $7 \mathrm{~s}$ to record outputs from the thermo-mechanical system. Continuous records of all the outputs were obtained during spindle activity (the table was positioned in the measuring location for the entire duration). The only interruption was second gear engagement in transmission between the motor and spindle as indicated in Fig 7. Measurement of both thermal source activities (spindle and table rotations) without interruption and processing of valuable information related to their interaction is subjected to analysis in subsequent paragraphs.

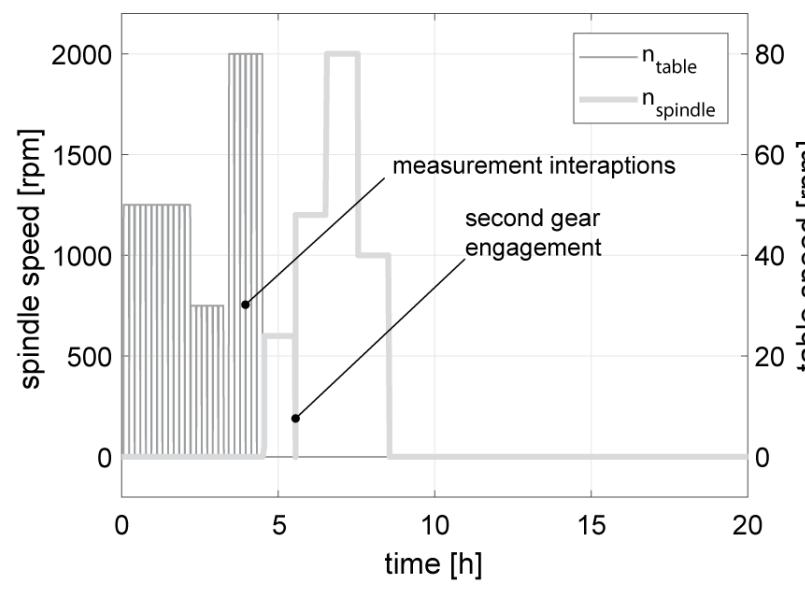

Fig. 7: Verification test set-up.

The behaviour of temperature inputs into the thermomechanical system in relative coordinates (spindle and table bearing temperatures and the temperature of the column representing changes in ambient temperature) during the verification test are depicted in Fig. 8. The influence of table cooling is not apparent during the verification test since both the calibration and verification were not carried out at the same time. The thermostatic regulation of coolant has been improved in meantime (the efficiency remained unchanged).

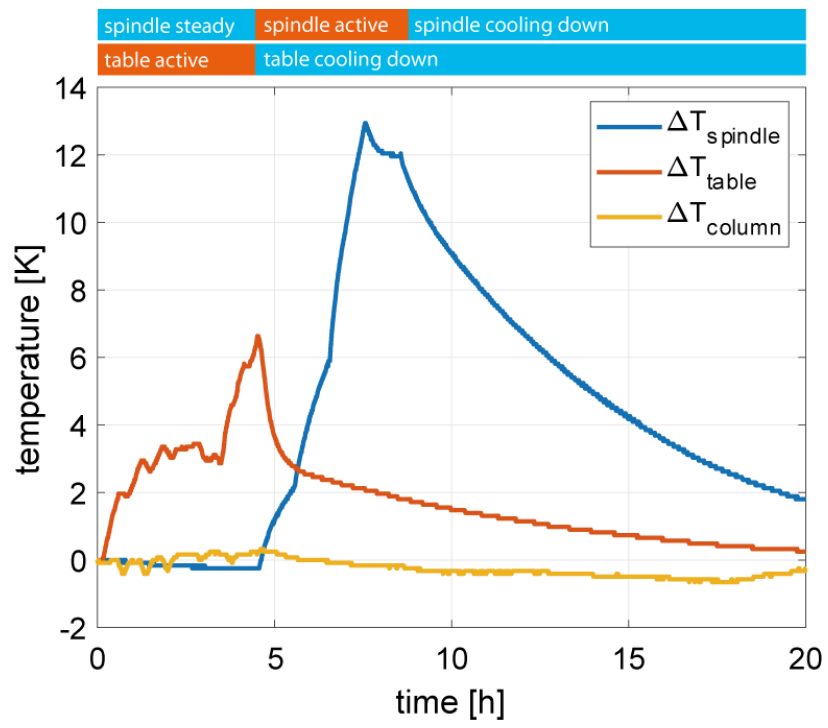

Fig. 8: Verification test conditions and thermo-mechanical system input behaviour.

Deformations measured in the $Z$ direction during the verification test are depicted in Fig. 9. As can be observed from this figure, the difference between the measured deformations in the table-TCP $\left(Z_{\mathrm{TCP}}\right)$ and table-frame $\left(Z_{\mathrm{D}=0}\right)$ positions is relatively small. Heat generated by spindle rotation influences a tool (a mandrel in this case) minimally and mostly passes into the body of the headstock. Therefore, it can be assumed that the compensation will be effective during finishing cutting conditions within milling (drilling, grinding) operations. Due to the symmetrical structure of the machine, negligible tilting of both the table and the headstock can be expected.

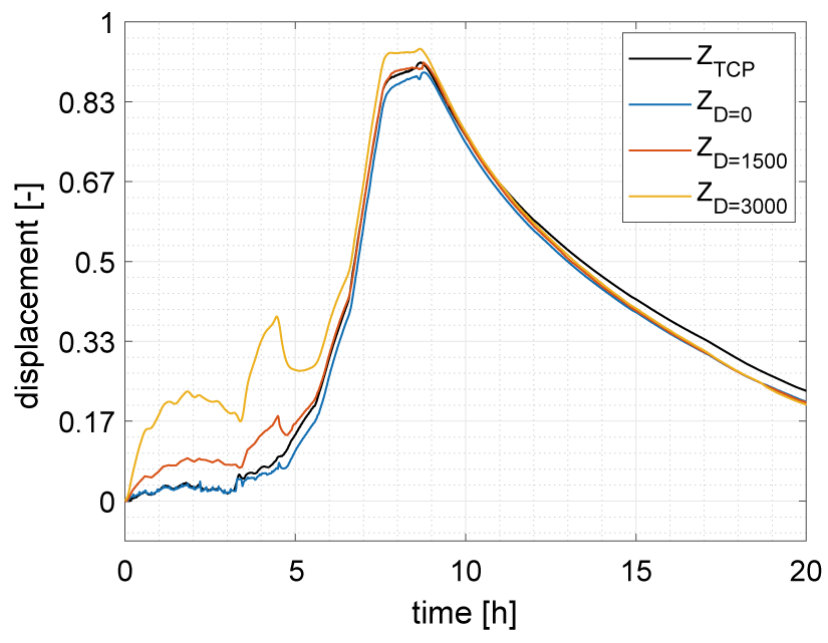

Fig. 9: Measured relevant outputs from the thermomechanical system during the verification test in TCP, $D=0, D=1500$ and $D=3000 \mathrm{~mm}$ measuring positions.

\subsection{Results}

The deformations measured in $D=3000 \mathrm{~mm}$ after compensation (residual error after TF compensation model application $\left.Z_{\mathrm{D}=3000}-Z_{\text {coupled }}\right)$ during the verification test in the 
$Z$ direction are depicted in Fig. 10, along with information regarding the uncompensated state $Z_{\mathrm{D}=3000}$. The developed model, per equation (8), can be divided in two parts (submodels). The first submodel describes the spindle speed influence $Z_{\text {milling; }}$ the second characterises the table rotation impact $Z_{\text {turning. }}$. The gain factor $g$ dependent on the workpiece clamping diameter was set-up to 2.2 for $D=3000 \mathrm{~mm}$ [Mareš2 2020]. The approximation model analysis of the contribution of deformational elements in the $Z$ direction thermal error compensation during the verification test is also depicted in Fig. 10.

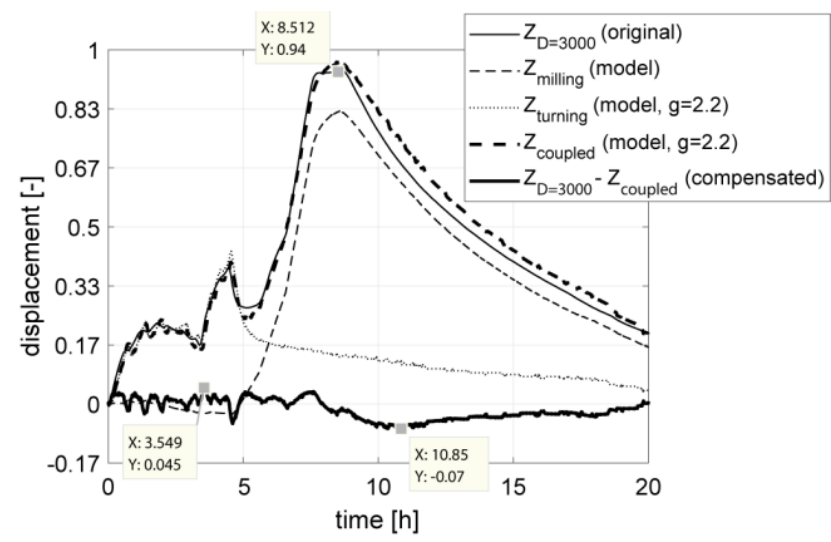

Fig. 10: Measured and simulated outputs from the thermomechanical system in $D=3000 \mathrm{~mm}$ measuring position and analysis of thermal error compensation during the verification test.

The fit value attained by model form equation (8) application during the verification test is $87 \%$ in the discussed measuring position $\mathrm{D}=3000 \mathrm{~mm}$. Expressed by peak-to-peak value, the original deformation state of the MT was reduced 6 -fold by compensation model application.

The MT deformation state in all measuring positions (TCP, 0,1500 and $3000 \mathrm{~mm}$ ) after application of the stand-alone position-independent submodel approximating influence of spindle rotation (equation (5)) is shown in Fig. 11.

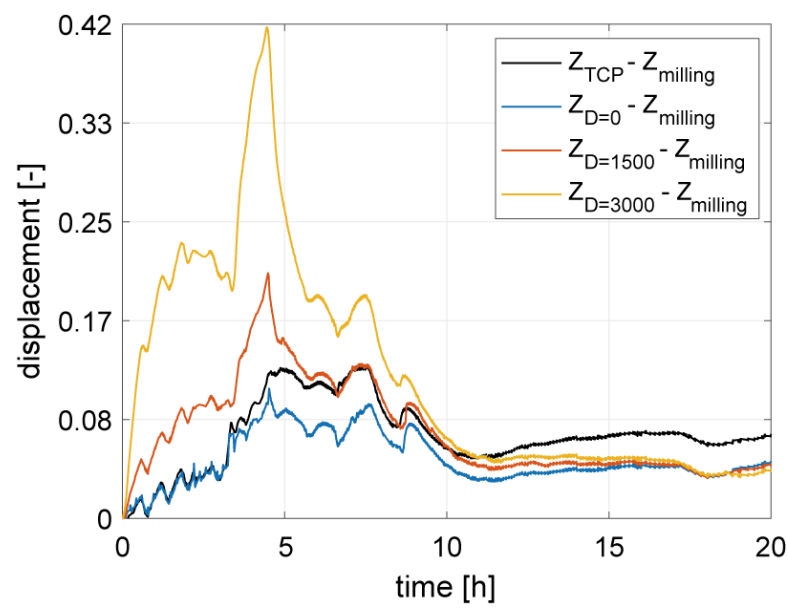

Fig. 11: Residual thermal errors in all measuring positions after application of the compensation model for spindle speed influence during the verification test.

The MT deformation state in all measuring positions after application of the combined model considering turning and milling operations (equation (8)) is shown in Fig. 12. The values of gain factor $g$ dependent on the workpiece clamping diameter are listed in the chart legend.

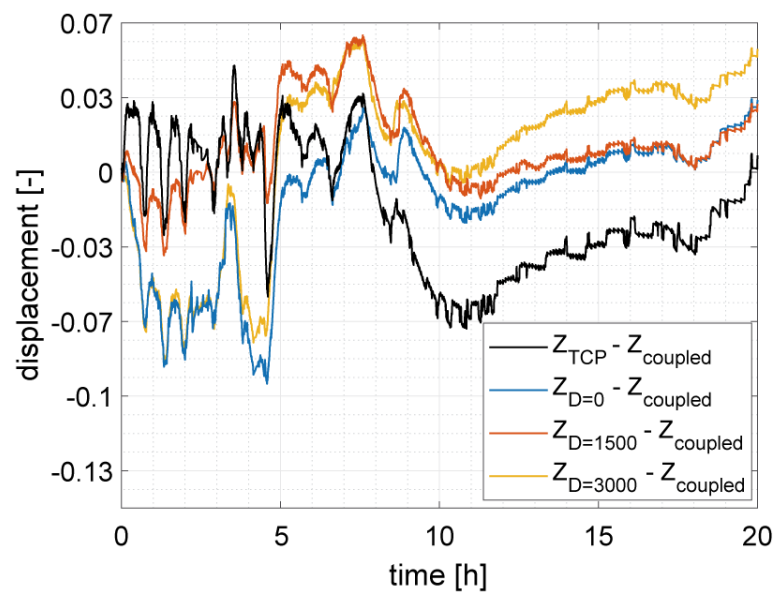

Fig. 12: Residual thermal errors in all measuring positions after application of the compensation model considering turning and milling operations during the verification test.

The average fit value after compensation in all measuring positions is equal to $88 \%$. At least a 6 -fold (up to 9 -fold) refinement of the original MT thermal error state caused by spindle and table variable activity was achieved by compensation model application.

The following table summarises the approximation quality achieved by the thermal error compensation model considering MT's capability of turning and milling operations from equation (8) application from both points of view: global (by fit value) and local (by the peak-to-peak method) in all examined measuring positions (TCP, 0, 1500 and $3000 \mathrm{~mm}$ ).

Tab. 3: Results of the compensation model considering MTs capability of turning and milling operations during the verification test.

\begin{tabular}{cccc}
\hline \multicolumn{4}{c}{ Measuring position [mm] } \\
\hline TCP & $\mathrm{D}=0$ & $\mathrm{D}=1500$ & $\mathrm{D}=3000$ \\
& \multicolumn{2}{c}{ fit [\%] } \\
82 & 90 & 92 & 87 \\
& \multicolumn{2}{c}{ Peak-to-peak [-] (-fold) } \\
6 & 7 & 9 & 8 \\
\hline
\end{tabular}

\section{SUMMARY}

The main objective of the scientific investigation presented in this article is enhancement of MT accuracy through minimisation of thermal errors and an evaluation of implemented (directly in the MT control system) compensation model regarding MT's capability of turning and milling operations. The role of the main thermal source, elimination of its influence on typical MT operations (turning, milling), workpiece clamping diameter consideration and a minimum increase of MT costs constitute basic requirements placed on specific compensation methods, and TFs seem to be a suitable apparatus.

The tested machine was a specialised vertical turning lathe capable of full-fledged turning and milling operations. All experiments were carried out under specific conditions: no cutting process was involved and they were performed along one MT axis configuration. The developed compensation model approximates undesirable thermal errors caused by spindle and rotary table activities. Compensation was taken into account for linear 
deformations in the $Z$ direction and along the entire table diameter. Measurements were realised with the help of a newly developed measuring fixture extended to the thermal stable frame enabling continuous measurement of spindle and table activity impact on the overall MT thermal error without interruption. The approximation quality of the model based on TFs was compared to the uncompensated MT state with an average result of $87 \%$ MT thermo-mechanical behaviour improvement. A comparison of the original and compensated MT thermal error states in the examined $Z$ direction is visible in Fig. 13.

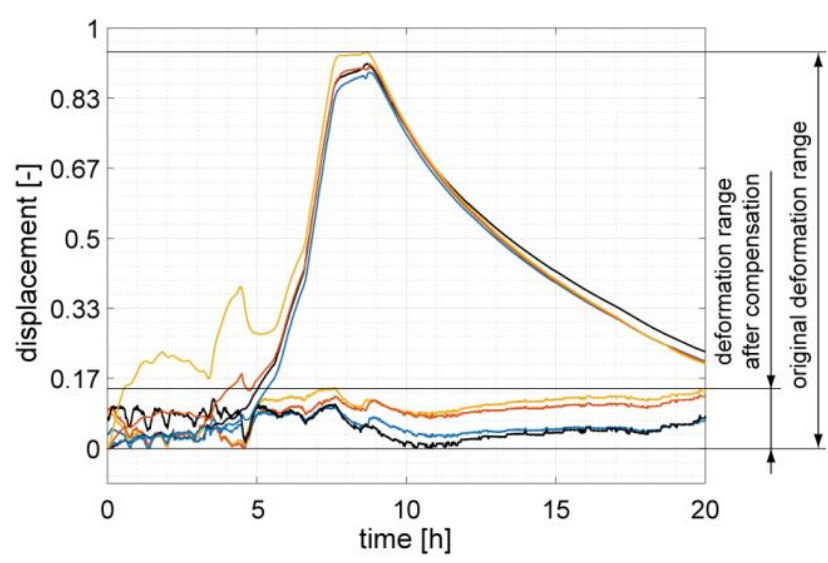

Fig. 13: Comparison of target machine accuracy with and without compensation during the verification test.

Relatively to the table impact, a high thermal error with long time constant caused by spindle rotation was observed. This is a result of lack of spindle cooling which can lead to spindle seizure. This issue is solved in a new generation of the target machine.

A critical point of any compensation model development is selection of suitable inputs into the thermo-mechanical system. From the perspective of modern trends in MT control, choosing a modelling approach with a model structure open to extensions of uncalibrated inputs and easy modification seems crucial. Compensation models will become a necessary part of smart solutions for intelligent MT and manufacturing. Such solutions rest on an assumption of long term stability and should have attributes suitable for machine learning principles. Compensation models have to be verified in real industrial conditions to guarantee their practical applicability. An evaluation of model transferability between machines of the same type and size and machines of the same type but of different sizes, components (e.g. spindle unit and spindle cooling system), and constructions (e.g. one-column structure) will be the focus of the recent and follow-up research.

\section{REFERENCES}

[Hernandez-Becerro 2018] Hernández-Becerro, P., et al., Model order reduction of thermal models of machine tools with varying boundary conditions. In: CIRP-sponsored 1st Conference on Thermal Issues in Machine Tools, Dresden, Germany, 21-23 March 2018.

[Blaser 2019] Blaser, P., et al., Long-term thermal compensation of 5-axis machine tools due to thermal adaptive learning control, MM Science Journal, 2019, pp 3164-3171. Available online: https://www.mmscience.eu/en [Brecher 2004] Brecher, C, et al., Compensation of thermoelastic machine tool deformation based on control internal data. CIRP Annals - Manufacturing Technology, 2004, Vol. 53, No. 1, pp 299-304.
[Grossmann 2015] Grossmann, K., et. al., Thermoenergetic design of machine tools: a systemic approach to solve the conflict between power efficiency, accuracy and productivity demonstrated at the example of machining production, Lecture Notes in Production Engineering, Springer. 2015, p. 260. ISBN 978-3-319-12624-1.

[Hellmich 2018] Hellmich, A.,et al., 2018, Methods for analyzing and optimizing the fluidic tempering of machine tool beds of high performance concrete. In: CIRPsponsored 1st Conference on Thermal Issues in Machine Tools, Dresden, Germany, 21-23 March 2018.

[ISO 230-3 2007] ISO 230-3 Test code for machine tools Part 3: Determination of thermal effects. International Organization for Standardization ISO, Geneva, Switzerland, 2007, p. 44.

[Li 2008] Li, J.W., et al., Thermal-error modeling for complex physical systems: the-state-of-arts review, Int J Adv Manuf Technol, 2009, Vol. 42, pp 168-179.

[Ljung 2020] Ljung, L. System Identification Toolbox TM User's Guide. The MathWorks, 2015. https://ch.mathworks.com/help/pdf doc/ident/ident ug.pdf

[Mares1 2020] Mares, M., et al., Thermal error minimization of a turning-milling center with respect to its multifunctionality. Int. J. of Automation Technology, Vol.14, No.3, 2020, pp 475-483.

[Mareš2 2020] Mareš, M., et al., Enhancement of vertical turning lathe accuracy by minimising thermal errors depending on rotary table activity and workpiece clamping diameter. Proceedings of the euspen's Special Interest Group: Thermal Issues, Aachen, Germany, 26-27 February 2020, pp 120-123.

[Mayr 2012] Mayr, J., et al., Thermal issues in machine tools. CIRP Annals - Manufacturing Technology, 2012, Vol. 61, No. 2, pp 771-791.

[Mayr 2018] Mayr, J., et al., An Adaptive Self-Learning Compensation Approach for Thermal Errors on 5-Axis Machine Tools Handling an Arbitrary Set of Sample Rates, CIRP Annals - Manufacturing Technology, 2018, Vol. 67, pp 551-554.

[Mize 2000] Mize, C., et al., Neural network thermal error compensation of a machining center, Prec. Eng., 24/4, 2000, pp 338-346.

[Moriwaki 2008] Moriwaki, T., "Multi-functional machine tool," CIRP Annals -Man Tech., 2008, Vol.57, No.2, pp 736749 .

[Naumann 2018] Naumann, C., et al., Hybrid correction of thermal errors using temperature and deformation sensors. In: CIRP-sponsored 1st Conference on Thermal Issues in Machine Tools, Dresden, Germany, 21-23 March 2018.

[Srinivas 2017] Srinivas, N. G., et al., Optimization of high speed machine tool spindle to minimize thermal distortion, Procedia CIRP 58, 2017; pp 457-462.

[Zimmermann 2020] Zimmermann, N., et al., Extended discrete R-Test as on-machine measurement cycle to separate the thermal errors in Z-direction. Proceedings of the euspen's Special Interest Group: Thermal Issues, Aachen, Germany, 26-27 February 2020, pp 21-24. 